\title{
Advances and Challenges of Corrosion and Topology Detection of Grounding Grid
}

\author{
Zhihong Fu ${ }^{1, *}$, Xiujuan Wang ${ }^{1, *}$, Qian Wang ${ }^{2}$, Xiaobin $\mathrm{Xu}^{1}$, Nengyi $\mathrm{Fu}^{3}$ and Shanqiang Qin ${ }^{1}$ \\ 1 School of Electrical Engineering, State Key Laboratory of Power Transmission Equipment and System \\ Security, Chongqing University, Chongqing 400044, China; xuxiaobin2017@cqu.edu.cn (X.X.); \\ shqiangqin@cqu.edu.cn (S.Q.) \\ 2 Electric Power Research Institute of Chongqing, Chongqing 401120, China; wangq239@163.com \\ 3 Depatrment of Geophysics, Colorado School of Mines, Golden, CO 80401, USA; \\ nengyifu@mymail.mines.edu \\ * Correspondence: fuzhihong@cqu.edu.cn (Z.F.); 20111101002@cqu.edu.cn (X.W.); \\ Tel.: +86-130-6235-2738 (Z.F.)
}

Received: 11 April 2019; Accepted: 30 May 2019; Published: 3 June 2019

\begin{abstract}
The grounding device plays performs the role of releasing a lightning current and a fault current in the power system, and the corrosion of the conductor will cause damage to the grounding body, which threatens the safe operation of the power system. The grounding grid corrosion detection technology and equipment guarantee the safe operation of the power system. This paper discusses the research status of grounding corrosion and topological detection in detail and introduces the basic principles, research difficulties and existing problems of the methods such as the electric network method, electromagnetic field method, electrochemical method, ultrasonic detection method and electromagnetic imaging method. The methods of electromagnetic imaging and time difference positioning proposed in recent years have been also discussed in detail. The paper points out that the application of grounding grid corrosion detection distance engineering still faces great challenges and that multi-disciplinary, multi-information fusion, new sensing technology, big data platforms and intelligent computing will be the trends to follow in research on grounding grid fault, corrosion detection and life prediction.
\end{abstract}

Keywords: grounding grid; metal corrosion; topology detection; corrosion detection; nondestructive testing

\section{Introduction}

The grounding engineering of power systems mainly includes substation grounding, tower grounding, and a high-voltage direct current grounding rod. A major purpose of grounding engineering is to ensure surface point stability and to reinforce the safety of personnel and equipment in the event of a lightning striking or massive amount of current discharged into the ground due to the failure of a power system. However, due to the fact that corrosion will inevitably occur in power systems at the subsurface level, this has become a potential safety hazard of grounding systems. Stray current corrosion, bacterial corrosion, and electrochemical corrosion are the main causes of grounding body corrosion [1,2]. Therefore, the diagnose of grounding body corrosion is one of the major challenges in the evaluation of grounding system conditions [3]. In addition, the detection of subsurface power system involves grounding network topology and grounding impedance detection. Grounding impedance detection technology and equipment are fairly developed [4]. Due to the high difficulty of the grounding body corrosion and topology detection, more advanced technology and equipment are required for such operations. 
Research concerning grounding body corrosion detection technology has been conducted for decades, yet it remains difficult to put theories into practice at a large scale. Copper and flat steel are commonly used as grounding conductors. Although copper has stronger corrosion resistance than flat steel, they both have been buried underground for a long time which can be up to more than 30 or 40 years. Thus, their possibility of being corroded cannot be ignored. In China, flat steel is mainly used as a grounding material, but it can be easily corroded. The research of corrosion detection is extensive. Corrosion detection technology of a substation grounding grid mainly includes the network method [5-26], electromagnetic field method [27-35], and electrochemical method [3,36-40]. In recent years, emerging technologies, such as ultrasonic testing [41,42], electromagnetic pulse time difference location [43] and electromagnetic imaging [44-49], have been considered for corrosion detection. Ultrahigh-voltage DC transmission has developed intensively in recent decades, and DC grounding corrosion is a severe challenge. When single pole earth return is used, the carbon steel corrodes rapidly. Thus, grounding corrosion has become the most difficult problem to solve in DC transmission projects. However, the depth, structure, and operation of grounding rods are quite different from those of grounding grids of substation. To our knowledge, no study has been conducted on the diagnosis of grounding rod corrosion. With the rapid development of new energy power generation, the corrosion of grounding bodies caused by a DC stray current in photovoltaic power plants is a new problem that is rarely discussed at present. Charalambos et al. [50] discussed a method of carrying out evaluations for grounding body corrosion caused by DC stray current in photovoltaic power plants. Because the grounding body is buried underground, it is concealed, and the degree of corrosion is difficult to detect directly. At present, evaluating grounding body corrosion mainly depends on site excavation, which is extremely low cost-efficient. It is also restricted by operating conditions. Therefore, the trenchless diagnosis and evaluation technology of grounding body corrosion is demanded.

Recently, grounding grid topology diagnosis technology has come to play an increasingly significant role. In grounding network fault detection and state assessment, accurate drawings of the grounding network are needed. However, in practical projects, old drawings of grounding networks with long a service life are missing or defective due to reconstruction and expansion. Intertwined old and new grids result in a large deviation between the drawing and the actual layout of grounding network, which contributes to the difficulty of fault diagnosis and state assessment of a grounding network. For the grounding project of newly built substations, effective detection methods to judge whether the grounding grid is constructed strictly according to the design drawings are not well-developed. For the fault diagnosis methods of grounding grids such as network method, determining the topological structure of grounding grids in order to establish the diagnosis equation is necessary. Therefore, a considerable amount of attention has been paid to the diagnosis technology of grounding grid topology. Accordingly, the electromagnetic field method, transient electromagnetic method (TEM), and some image processing technologies have been developed in recent years.

\section{Corrosion Detection Method of Substation Grounding Grid}

According to the detection principle, grounding grid corrosion diagnosis methods can be generalized as the electrical network method, electromagnetic field method, electrochemical method, ultrasonic detection method, and electromagnetic imaging method.

\subsection{Electric Network Method}

The corrosion or fracture of nodes can be obtained by taking the resistance of ground network conductor as the fault parameter, combining the diagnosis theory with the network theory, adopting the non-linear optimization method, and comparing with the original data. The electric network method can be divided into port resistance method, sensitivity analysis method, and improved sensitivity analysis method. 


\subsubsection{Port Resistance Method}

The basic principle of the port resistance method based on Telegen's theorem is described in $[5,6]$. It was first proposed by Huang Yong and He Xingbai of Chongqing University in the early 1990s. The principle of the port resistance method is shown in Figure 1. Grounding grids are usually formed by the interconnection of horizontal equalizing conductors. They are buried underground. Single grids are usually rectangular, and the materials are ordinary galvanized flat steel, round steel, or flat copper. Ignoring the influence of soil and other factors, the grounding conductor is regarded as an equivalent of resistors, and the points connecting the down-line and the grid are assumed to be located at the nodes of the grid without any deviation. The grounding conductor network after the completion of grounding grid laying is set to N. According to the structural drawing, and the size and material of the laying conductor, the complete conductor resistance values of each section can be obtained. After using the grounding grid for a long time, the resistance parameters of the grounding conductor are changed due to corrosion. At this time, the network is set to $N^{\prime}$. The network $N$ and $N^{\prime}$ have the same topological structure and can be applied to Tellegen's theorem. By injecting current $I_{0}$ between accessible nodes $i$ and $j$ of network $N$ and $N^{\prime}$ and measuring the port voltage $U_{i j}$, we can calculate the port resistance $R_{i j}$ and $R_{i j}$ of network $N$ and $N^{\prime}$ and then obtain the change of port resistance $\Delta R_{i j}$. If the network $N$ and $N^{\prime}$ have m group accessible nodes, the injected nodes can be replaced in turn, and the m group port resistance can be obtained. According to Kirchhoff's current law (KCL), Kirchhoff's voltage law (KVL), and Tellegen's theorem, the diagnostic equation of the electric network method can be deduced.

$$
\left\{\begin{array}{l}
\Delta R_{i j\{1}=\sum_{k=1}^{b} \Delta R_{k} I_{k\{1} I_{k\{1}^{\prime} / I_{0}^{2} \\
\Delta R_{i j\{2}=\sum_{k=1}^{b} \Delta R_{k} I_{k\{2} I_{k\{2}^{\prime} / I_{0}^{2} \\
\cdots \\
\Delta R_{i j \mid m}=\sum_{k=1}^{b} \Delta R_{k} I_{k\{m} I_{k\{m}^{\prime} / I_{0}^{2}
\end{array}\right.
$$

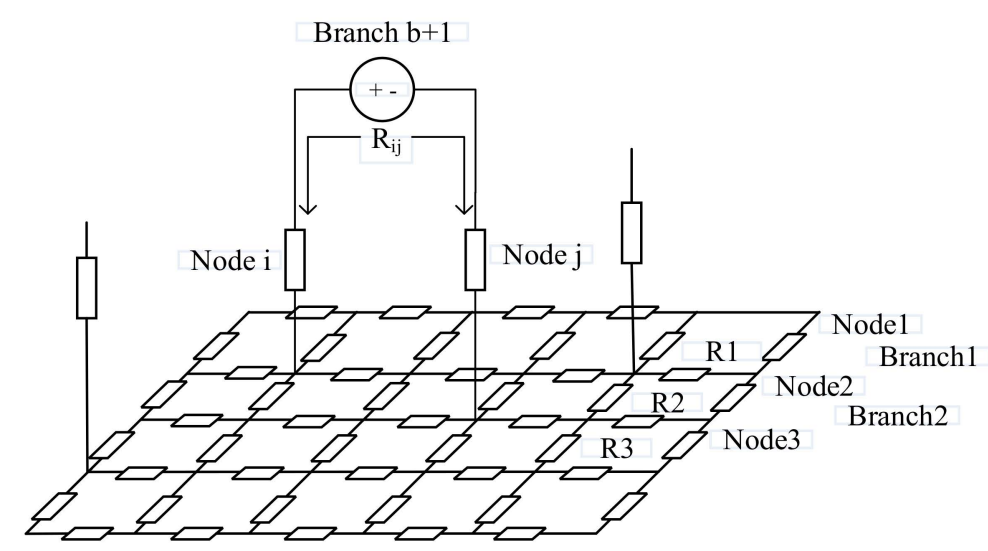

Figure 1. Schematic diagram of port resistance method [5].

In Formula (1), $I_{0}$ is the injection current of two accessible nodes, and $\Delta R_{i j}$ is the change of port resistance measured by injection current between two accessible nodes. $I_{k}$ is based on the current of each branch of network $N$ at the injection of $I_{0}$. All three quantities are known. $\Delta R_{k}$ is the variation of branch resistance before and after network corrosion, $I{ }^{\prime}{ }_{k}$ is the branch current of network $N^{\prime}$; thus, $\Delta R_{k}$ and $I \prime_{k}$ are unknown.

Formula (1) is a non-linear system of equations, and the number of equations (m) is usually less than the number of branches (b). The formula belongs to the undetermined system of equations and cannot be solved directly. Generally, an iterative algorithm is implemented to solve this equation. 
The effectiveness of port resistance method depends on the accuracy of port resistance measurements. The application of this method is restricted by instrumental error from measuring the small resistance value of grounding conductor and the influence of down-line resistance, contact resistance, and cable resistance.

\subsubsection{Sensitivity Analysis Method}

Chen [7-9] proposed a sensitivity analysis method to diagnose grounding grid corrosion. In this method, conductor resistance increment of the grounding grid is taken as the fault parameter, and the fault diagnosis equation is established by sensitivity analysis method. The principle of minimum energy and optimization technology are introduced into the solution of the fault diagnosis equation.

For grounding grids with $\mathrm{b}$ branches and $\mathrm{r}$ accessible nodes, a fault diagnosis Equation (2) is established.

$$
\Delta \mathrm{U}_{\mathrm{r}}=\mathrm{U}_{\mathrm{rb}} \cdot \mathrm{X}
$$

where $U_{\mathrm{rb}}$ is the sensitivity matrix and $U_{i j}$ represents the effect of branch $j$ conductor resistance on the potential of measuring node $i . \Delta \mathrm{U}_{\mathrm{r}}$ is the variation of node voltage before and after corrosion, which can be obtained by measurement. $\mathrm{X}$ is a $\mathrm{b}$-dimensional column vector, $x_{j}$ represents the multiplier of the conductor resistance increment in the $j$-th segment, which is to be determined. For the actual grounding grid, the fault diagnosis equation is undetermined because the number of accessible nodes $\mathrm{m}$ is always less than the number of branches $\mathrm{b}$. The sensitivity matrix is related to branch resistance; that is, the sensitivity matrix is not constant, so Formula (2) is a non-linear equation and is established only when resistance slightly changes.

$$
\operatorname{Min} \mathrm{P}=\sum_{i=1}^{b} I_{j}^{* 2} R_{j}^{*}
$$

The application of optimization theory to the solution of the undetermined equation can solve the problem of calculating the actual resistance of conductors in each segment of the grounding grid.

\subsubsection{Improved Sensitivity Analysis Method}

Because the sensitivity analysis method only makes use of a very limited number of equations and does not take non-linearity into consideration, the resistance of each conductor segment deviates greatly from the actual value. Liu [10] and Luo [11] have improved the sensitivity analysis method. They rotated the excitation position of the current source in the accessible node and measured the voltage of nodes at many locations when each excitation occurs. They made full use of the limited accessible nodes and greatly expanded the number of equations.

The diagnostic equation of the electric network method is a set of undetermined or overdetermined non-linear equations, and the optimization algorithm is a research hotspot. The simplex method $[7,8]$, TABU search algorithm [12], support vector regression [13], differential evolution algorithm [14], constrained total least squares algorithm [15], genetic algorithm [16], and dynamic chaotic PSO [17] have all been applied. Yang [18] proposed Tikhonov regularization on the basis of the least square method in order to reduce the ill-conditioning of non-linear equations, and used the L-curve method to identify grounding grid faults.

Fault diagnosis for large-scale networks requires not only large size matrix operations but measurement errors and mutual-interference. Tearing and block testing methods [19-21] have been developed. In the partial fault diagnosis of grounding grid, only the relevant parts can be tested, which can reduce the testing workload and effectively improve the fault diagnosis efficiency.

Liu [22] and Luo [23] analyzed the branch corrosion measurability of the grounding grid. They eliminated inaccessible nodes by network transformation and carried out inversions to determine the measurability of each branch gradually. The testability of fault diagnosis of the grounding grid is 
predictable, which has guiding significance for optimizing the test scheme and making scientific use of the diagnosis results.

The substation site is complex, and the practical application of the network method involves many obstacles. The influence of down-line offset [24], portal frame [25], and cable trench [26] is a difficult problem to be solved urgently in the power network method; thus, further research is necessary.

The electric network method has been put forward for nearly 30 years, nevertheless, the testing equipment and implementation methods are not sufficiently practical, as the result, their popularization are obstructed. The network method needs to be developed in more practical manners to determine the topological structure of the grounding network. Establishing an accurate grounding network model is difficult because of irregular construction, lack of drawings, inconsistency of construction and drawings, incomplete grounding network, and connection between the new grounding network and the old one. Moreover, the selection principle of down-line is difficult to determine, and the grounding conductor resistance is very small. In order to suppress strong electromagnetic interference, high power supply is required. There are factors such as the deviation of the positions of the accessible nodes, measuring the resistance of the wire, contact resistance, cable trench and surrounding equipment, which will affect the measurement results. In addition, it has large measurement workload, difficult data management, and low detection efficiency. Hence, operators should have a high technical threshold and limited practicability.

\subsection{Surface Electromagnetic Field Method}

The surface electromagnetic field method carries out data acquisition from the surface magnetic field, analyzes surface magnetic field characteristics and determines the distribution and fault of grounding grid conductors. Two kinds of grounding grid methods are present: injected and non-injected.

The non-injected type uses the magnetic field induced by the ground current in the ground network to directly measure and analyze its characteristics for fault diagnosis. Gomes et al. [27] designed the surface electromagnetic field detection system and developed a fault diagnosis program on the basis of the finite difference method. This method can test the surface potential, test the distribution of grounding grid, and evaluate the unbalanced current of transformer and the corrosion degree of grounding grid. The non-injected surface electromagnetic field method requires the presence of grid current from power station, which is simple and convenient to set up. However, this method is subject to the change of in situ current and therefore is less used for research and applications.

The injected surface electromagnetic field method was proposed by Dawalibi [28] in 1986 to measure the electromagnetic field distribution on the ground surface which is induced by current injected into the down-line of grounding grids. By comparing data with the theoretical model, local electromagnetic field changes were obtained to determine the locations of grounding grid faults. Dawalibi analyzed the impact of injection mode (location and number of injection points), frequency, and network structure on the diagnosis. Since then, many scholars have studied various forms of injected current, namely DC, alternating current (AC), square wave, and HF. Zhang et al. [29] applied high-frequency $\mathrm{AC}$ current injection (up to $1 \mathrm{MHz}$ ) to diagnose the grounding grid breakpoint on the basis of the leakage current. The higher the injection current frequency, the better the diagnostic resolution. However, developing a $1 \mathrm{MHz}$ high-frequency current source is difficult. The influence of displacement current should also be considered. Cui and Liu adopted a $379 \mathrm{~Hz}$ AC injection current to determine the corrosion degree of ground grid by measuring the drop in magnetic field amplitude [30]. A low-frequency current source reduces the difficulty of power supply design. $\mathrm{He}$ [31] injected $50 \mathrm{~Hz}$ and $10 \mathrm{~A}$ current into high-speed railway grounding systems and realized the corrosion diagnosis of the grounding conductor through the sur face magnetic field. On the basis of the geophysical frequency-domain detection and dual-frequency induced polarization method, the dispersion characteristics of soil and proposed injecting double-frequency square wave current could be realized [32]. The square wave current has broadband characteristics, and some frequency 
points may amplify the corrosion information of the grounding grid to improve the detection effect of the surface magnetic field method. Qamar $[33,34]$ used the differential operation to amplify the characteristic signal (the third-order differential of the surface magnetic field), locate the grounding grid conductor with peak value, realize grounding network topology detection, and determine the flat steel fracture on the basis of the missing third-order differential peak of the surface magnetic field. However, the differential operation will amplify the noise, which is not practical under the presence of strong electromagnetic interference in the substation. In order to reduce measurement workload, Yang [35] measured the magnetic induction intensity of some measuring points above the grounding grid of substation, established the inverse problem of the grounding grid magnetic field, solved the inverse problem using the reconstruction method based on magnetic detection electrical impedance tomography (MDEIT) method, reconstructed the magnetic field model above the grounding grid, and realized the corrosion fault diagnosis of the grounding grid.

The electromagnetic field method is much simpler than the electric network method in terms of working mode and fault diagnosis characteristic analysis. Moreover, the operability is significantly improved, but the method still has many problems: The noise from the substation is very large, which will affect the judgment accuracy. Moreover, the injection current position is difficult to normalize as it is easily affected by the injection down-line. In addition, the layout of the down-line has not yet been thoroughly investigated. Given the soil dispersion characteristics, the optimal injection frequency and waveform shape are not clear. Moreover, research on the electromagnetic field distribution in the case of the uneven distribution of grounding conductors and complex soil resistivity is still underdeveloped, and the transformer, cable trench, bracket, and other electrical equipment can be easily influenced.

\subsection{Electrochemical Method}

The electrochemical method determines the corrosion degree or rate of metals by measuring the electrochemical characteristics of the corrosion system between metals and soil, including potentiometry, electrochemical noise, linear polarization, AC impedance, and electrostatic method. The electrochemical method has successfully been applied to detect corrosion of pipelines, concrete reinforcing bars, and metal components. However, the corrosion detection of groundwork has only begun in recent years. The detection targets of the electric network and surface electromagnetic field methods are the corrosion degree of the grounding grid. The electrochemical method mainly detects the corrosion rate of metals. Therefore, it can be used to evaluate the state of the grounding grid and predict its service life span. The electrochemical method has been widely used in the study of corrosion resistance of grounding conductor materials [3,36,37]; by contrast, the corrosion state detection in grounding grid is seldom used. Han et al. [38] proposed a constant current step method to detect the corrosion of the grounding grid and obtained the polarization resistance value by analyzing the charging curve to reflect the corrosion of the grounding grid at the test location. Yang and Peng [39] used the quasi-steady-state measurement of step voltage excitation to obtain the corrosion rate of the ground conductor.

The physical and chemical significance of electrochemical measurement method is clear and it reveals the nature of corrosion. From this point of view, it is a research direction with development prospects. However, electrochemical method is not yet mature and the problems with it focus on:

1. Reliability of corrosion rate detection. This problem has three causes: (1) The influence of AC and DC dispersion. (2) The conductor dispersion is not uniform. The dispersion is affected by the location of the injection point and the distribution of the geoelectric field, and the dispersion of conductors in each segment is extremely uneven. (3) Sensor installation brings influences to the original soil environment. The electrochemical method can only detect the corrosion rate of a test point, and the measurement results are discrete in time and location.

2. Sensor current limiting problem. As a whole, the ground grid is a working electrode. The polarization current is not confined to the measured conductor. The uncertainty of the polarized ground network area makes the corrosion rate impossible to calculate. Han et al. [38] designed a small hole current-limiting device for corrosion detection of the grounding grid. The basic 
principle of this device is to use the electric field to guide the focusing and directional flow of the polarization current in soil, so as to achieve current limiting. This method has been applied in concrete corrosion monitoring [40].

3. Strong electromagnetic noise. Strong electromagnetic interference is present in power plants, substations and transmission lines, ground grid dispersion, and stray current effects. There are methods such as wavelet denoising, but the polarization current is on the order of $\mu \mathrm{A}$. Compared with ground grid and stray current, it is far less than an order of magnitude, and the signal feasibility is not high.

4. Poor accuracy of corrosion assessment. The electrochemical method usually obtains the corrosion rate by measuring the corrosion current and then estimates the corrosion degree by integral. It is an indirect measurement method. The integral calculation produces cumulative error.

In addition, the electrochemical method for the grounding grid is a microscopic measurement. The measurement can only measure the corrosion rate of the measurement point, but it is impossible to judge the location of the breakpoint and cannot applied in the case of the reinforced concrete ground.

\subsection{Time Difference Location Method}

Time difference location is a newly proposed method to determine the defect location of grounding grids. This method involves ultrasonic detection and electromagnetic pulse time difference location (D-TDOA).

The ultrasonic detection method utilizes the uniform velocity propagation of ultrasonic waves to determine the reflection characteristics of defects, determines the defect location by calculating the time difference of the received reflected wave, estimates the defect magnitude according to the reflected wave amplitude, and detects the non-electromagnetic parameter by ultrasonic method [41,42]. This method is not sensitive to strong electromagnetic interference in power systems, which is its main merit. The overall response of the whole network is measured by the electric network method, but the transmission speed of the guided wave is slow. Early signal response conductor near-end corrosion information can eliminate the influence of near-grid conductor. However, the underground network is deeply buried. In addition, the directional problem caused by the mesh structure, influence of the flat steel solder joint, down-line and electrical equipment connection points, and influence of soil non-uniformity are difficult to solve for the ultrasonic guided wave detection method. A time difference location method (D-TDOA) was proposed to inject the Gauss pulse current and detect pulse transmission time with a broadband measurement sensor $(1 \mathrm{kHz}-1 \mathrm{GHz})$ [43]. The fault location accuracy is $10 \mathrm{~cm}$. This method has not been tested on site. Moreover, the output power is a huge problem because the excitation source frequency is very high.

\subsection{Electromagnetic Imaging}

In recent years, the research achievements in geophysical exploration, biomedical imaging, and other fields have brought vitality to the diagnosis of grounding grid corrosion, forming a branch of the electromagnetic imaging method.

TEM is a geophysical method. It establishes a pulsed magnetic field underground by sending current through a loop. During a field intermission, the secondary eddy current field is observed by a receiving coil, and the longitudinal resistivity profile is formed by inversion. TEM has the characteristics of strong penetration, high resolution, small volume effect, and non-contact measurement. It is mainly used in the field of geophysical exploration. Therefore, the author proposed the transient electromagnetic method for grounding grids (GG-TEM method) [44-46]. The basic principle of GG-TEM is shown in Figure 2. The transmitter outputs bipolar pulse current and excites the magnetic field through the coil. At the moment of a magnetic field turn-off, the secondary eddy current field is excited at the ground grid, and the eddy current is confined within the grounded grid and attenuated. The receiving coil measures the secondary eddy current magnetic field in the ground 
network intermittently in one pulse. The magnitude and attenuation rate of the secondary eddy current field are determined by the thickness of grounding grid conductor, electromagnetic characteristics of the material, and corrosion degree. The apparent resistivity imaging method is used to obtain the sectional map of the grounding grid. The corrosion degree of the grounding body can be analyzed by the apparent resistivity section characteristics.

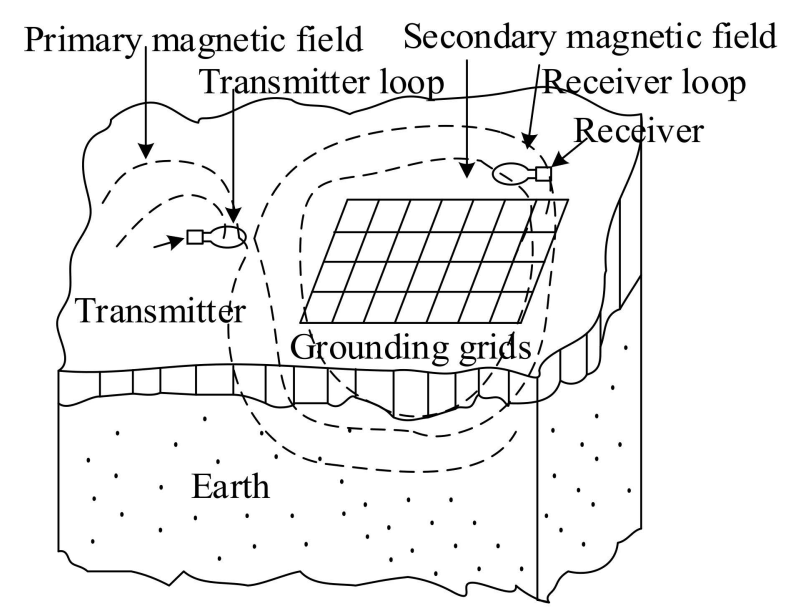

Figure 2. A Schematic field setup of transient electromagnetic method [46].

In 2016, we conducted experiments in the Wuhan Nanrui Grounding Network Experimental Field and achieved fairly ideal detection results (Figures $3-5$ ). The grounding grid, grid spacing, trench width, and depth were $24 \mathrm{~m} \times 24 \mathrm{~m}, 4 \mathrm{~m}, 0.4 \mathrm{~m}$, and $0.6 \mathrm{~m}$, respectively. The degree of corrosion was simulated by the thickness of the flat steel. The thinner the flat steel, the higher the corrosion degree. Four zones $(60 \times 6 \mathrm{~mm}(\mathrm{~A}), 40 \times 4 \mathrm{~mm}(\mathrm{~B}), 20 \times 3 \mathrm{~mm}(\mathrm{C})$, and $40 \times 4 \mathrm{~mm}$ flat steel (D)) were set, with four fracture ports (numbered $1,2,3$, and 4 ).

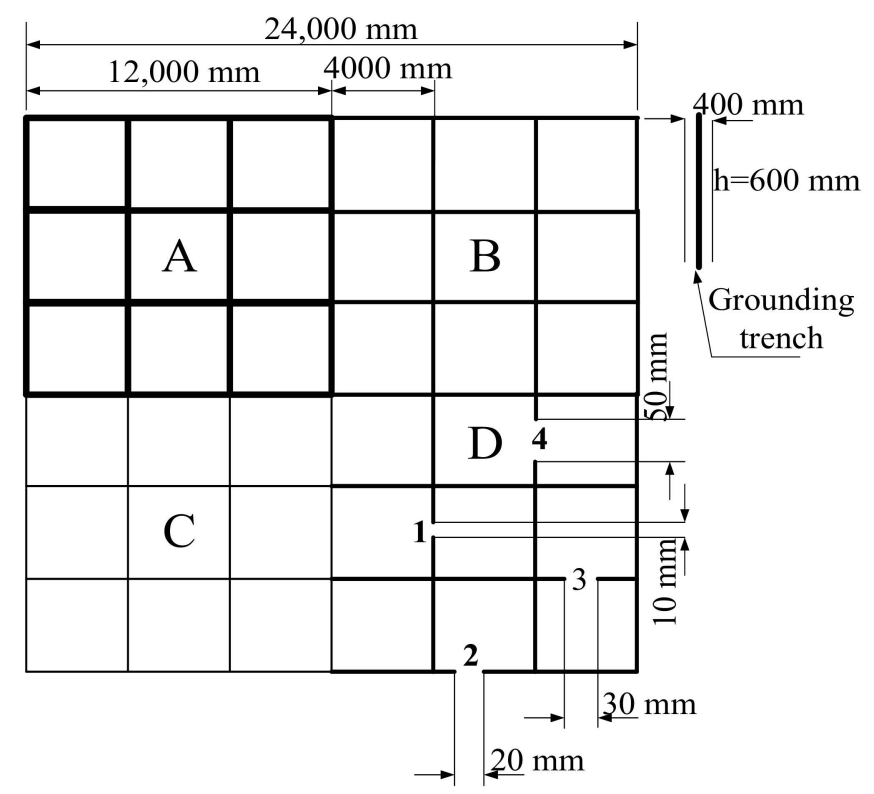

Figure 3. Wuhan Nanrui grounding grid experimental site. 


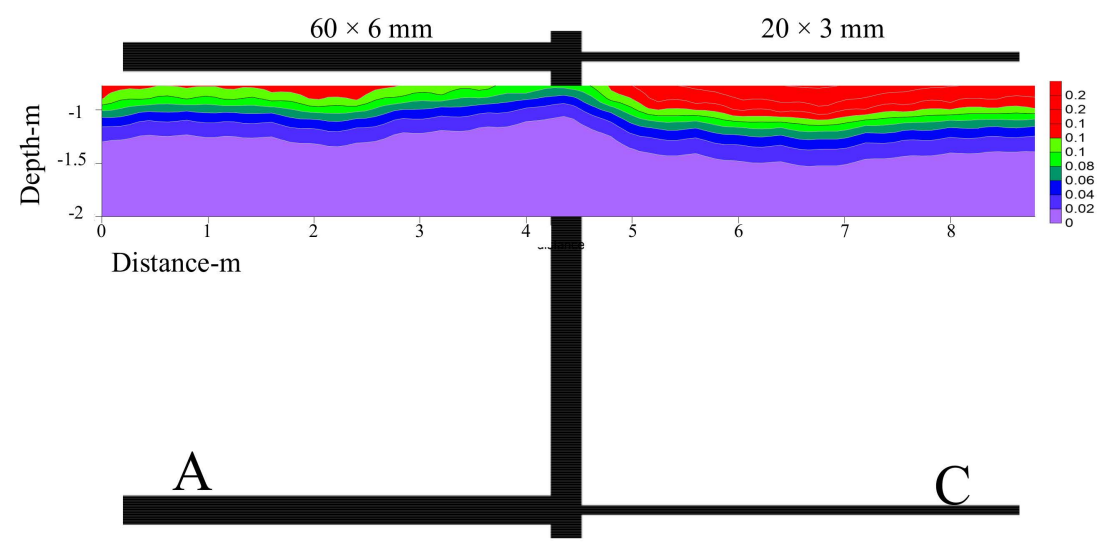

Figure 4. Apparent resistivity section of flat steel.

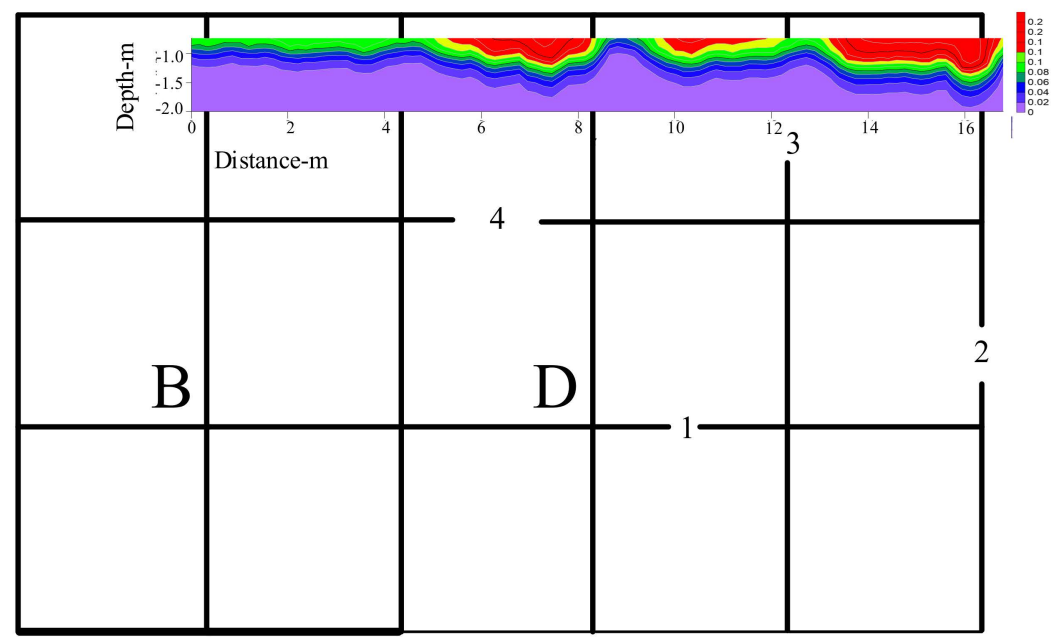

Figure 5. Apparent resistivity section with breakpoints.

Two lines were arranged to detect the imaging characteristics of the thickness and breakpoint of flat steel. In Figure 4, the measuring line passes along the flat steel through areas of different thickness $(60 \times 6 \mathrm{~mm}$ and $20 \times 3 \mathrm{~mm})$. As shown in the figure, the high-resistivity layer is thin, and the resistivity is low in the thick conductor section. In the thin conductor section, the high-resistivity layer is thicker, and the resistivity is higher. The electrical resistivity imaging characteristics coincide with the thickness of flat steel. Breakpoint detection: The line passes through the BD area, which has the same flat steel thickness $(40 \times 4 \mathrm{~mm})$, and the line passes through the breakpoint grids of 4,1 , and 2 . Figure 5 shows that the apparent grid resistivity of the three grids where the breakpoints are located is significantly higher than that of the intact grid. Moreover, the thickness of the high-resistivity layer is obvious, and the resistivity of the intact grid is low.

Compared with the surface electromagnetic field method, GG-TEM can solve the problem of current injection point of electromagnetic field method, normalization of injection point, primary field influence problem of electromagnetic induction method, and contact resistance problem and realize contactless measurement.

Yang et al. [47] proposed the grounding net imaging method of endogenous EIT. The 16-channel device mode and imaging principle of biomedical electrical impedance tomography were adopted. The amount of information was greatly increased, and the construction efficiency and diagnostic accuracy were improved. Wu [48] and Wang [49] proposed the radar imaging method, where the ultrawideband emission signal propagates through the soil medium to reach the grounding body and the echo signal is used to obtain the physical state of the grounding body. 
In recent years, the electromagnetic imaging method has become a hotspot in the research of grounding grid corrosion diagnosis. However, this method is limited by problems such as reinforced bar shielding of concrete pavement, cable trench, power equipment impact, and large calculation workload.

\section{Grounding Network Topology Detection Method}

The current injection method and magnetic field excitation method are the main detection methods of grounding grid topology structure.

\subsection{Current Injection Method}

The current injection method injects a sinusoidal current by selecting a suitable injection node, measures the magnetic field on the surface, and analyzes the magnetic field distribution characteristics to realize the grounding conductor positioning.

Yang [30] designed and developed a different frequency current supply system, mobile weak magnetic induction intensity measurement system, and corresponding software system. Qamar [33] proposed a grounding network topology detection method on the basis of the differential method and established a magnetic field shape function for describing the direction of the ground surface parallel or perpendicular to the grounding grid. This method uses the principal peak characteristic of the even derivative of horizontal component shape function and odd derivative of vertical component shape function to filter and differentiate the measured data of a square magnetic field in the grounding grid after injecting a current, so as to determine the topological structure of the grounding grid. However, the differential method is very sensitive to noise and measurement error. The detection path has a good effect perpendicular to the conductor direction, and the positioning result may have a large error when a large deviation from the vertical direction exists [30,33]. Fu et al. [51] proposed a grounding network topology detection method on the basis of wavelet edge detection technology. Wavelet edge detection effectively strips the magnetic gradient characteristics of each conductor segment from the background field, which greatly reduces the influence of magnetic field superposition on positioning accuracy. At the same time, the strong anti-interference ability of the wavelet transform suppresses the error introduced in the measurement and magnetic field fitting process and ensures the method's stability.

\subsection{Magnetic Field Excitation Method}

The magnetic field excitation method excites the grounded flat steel through the coil to generate the eddy current field (the secondary field), and the topology of the grounding network is obtained by detecting the eddy current field. The transient electromagnetic method [46] measures the net secondary field, and it has high detection accuracy. The topography of the grounding grid can be visually obtained using the apparent resistivity imaging technology, and the image is intuitive, but it has a large amount of calculation. In addition, the topology of the grounding grid can be obtained by analyzing the amplitude characteristic of the magnetic field which generated by the sinusoidal current. The method is simple in measurement and small in calculation, but the background field is included in the measurement result of the method, so the detection accuracy is relatively low.

\section{Challenges and Opportunities}

Although a variety of grounding grid corrosion and topological detection methods have been proposed, these methods are still immature, and commercial equipment and technical regulations are lacking. The following are challenges from a technical perspective:

1. Strong electromagnetic interference

The electromagnetic environment of the substation is very complicated, and the power frequency interference magnetic field of the substation operating state can reach more than $10 \mu \mathrm{T}$. The high-frequency transient interference caused by high-voltage switching action; large power frequency current from ground power equipment, such as transformers, knife gates, transformers, 
and gate brackets; power grounding current; harmonic interference; and various secondary and communication equipment will affect the normal operation of the detection instrument by means of spatial and conduction coupling.

The strong electromagnetic interference poses a serious threat to the electrical network method, surface electromagnetic field method, electrochemical method, and electromagnetic imaging method. By using an active source, the excitation source power is increased, and the signal-to-noise ratio is improved. Changing the frequency of the excitation current signal and using a frequency-selective amplification technique, such as notch, amplification, and filtering, can suppress the power frequency and avoid the main interference frequency.

2. Strong metal interference

The substation has a large number of metal facilities, and the connection of several important facilities to the grounding network brings great difficulties to the detection of the grounding grid. For example, a large number of underground metal pipes, cables, leads and cement roads with steel structures will have a great impact on the distribution of surface magnetic fields. This makes it difficult to distinguish the grounding grid information.

3. Corrosion detection of down-line

The grounding grid down conductor is a communication channel between the power equipment and the grounding network. The stray current density is large near the surface layer of the soil, and because of the direct contact of the air, it is susceptible to changes in air and soil humidity, so that the corrosion rate of the part of the grounding conductor is higher than that of the horizontal conductor of the grounding grid. Therefore, the down-line underground part is easily corroded. Moreover, diagnosing down-line corrosion is very difficult, and studies on the corrosion diagnosis of the down-line are lacking.

\section{Small target body of flat steel in grounding grid}

The common cross-sectional area of the grounding grid conductor is very small compared to other geophysical targets. The cross-sectional area of the grounding grid is much smaller than the length of the conductor, which brings great difficulty to the software simulation. For the electromagnetic induction method, because the conductor cross section is small, the eddy current response intensity and the influence range are small; for the ground penetrating radar, the reflection surface is too small, which brings great difficulty for detection.

\section{Multi-information fusion}

The electric network method, surface electromagnetic field method, time difference positioning method, and electromagnetic imaging method consider the physical characteristics of the grounding grid corrosion. However, these methods do not reveal the nature of corrosion and can only solve the problem of corrosion degree detection. The electrochemical method can detect the corrosion rate but not the degree of corrosion. Multi-information fusion [52], combined with multiple methods, is expected to simultaneously achieve corrosion degree and corrosion rate detection. Therefore, tapping the potential of existing methods, implementing two or more corrosion detection methods in one device, solving the problem of simultaneous detection of corrosion degree and corrosion rate, and improving the practicality of the detection method are future development directions.

6. Life prediction of grounding grid

With the development of power grid technology and the improvement of voltage levels, the safe operating life of grounding grids has begun to receive attention. It is expected to realize the life prediction of the grounding grid by detecting the corrosion degree of the grounding grid conductor, the corrosion rate, and the parameters of soil aeration, water content, salt content, $\mathrm{pH}$ value, soil resistivity, oxidation-reduction potential and soil temperature. Multidisciplinary intersection, multi-information 
fusion, new sensing technology, big data platform [53] and intelligent computing will certainly promote the maturity and application of grounding grid detection technology and life prediction. These bring new opportunities for research in the field of grounding grid testing.

Author Contributions: Formal analysis, Z.F. and X.W; Methodology, X.W. and X.X.; Project administration, Z.F., Q.W. and S.Q.; Software, S.Q.; Supervision, Q.W.; Validation, N.F.; Writing-original draft, Z.F. and X.W.; Writing-review \& editing, X.X. and N.F.

Funding: This research was funded by National Key R\&D Program of China, grant number 2017YFC0601804, and National Natural Science Foundation of China, grant number 1777017.

Acknowledgments: This work was supported in part by the National Key R\&D Program of China (No. 2017YFC0601804) and the National Natural Science Foundation of China (No. 51777017).

Conflicts of Interest: The authors declare no conflict of interest.

\section{References}

1. Schaefer, L.P. Electrical grounding systems and corrosion. Trans. Am. Inst. Electr. Eng. Part II Appl. Ind. 1955, 74, 75-83. [CrossRef]

2. Rajan, S.; Venugopalan, S.I. Corrosion and grounding systems. IEEE Ind. Appl. 1977, IA-13, $297-306$. [CrossRef]

3. Lawson, V.R. Problems and detection of line anchor and substation ground grid corrosion. IEEE Trans. Ind. Appl. 1988, 24, 25-32. [CrossRef]

4. Tong, X.F.; Zheng, Z.H.; Tan, B.; Lu, H.Y.; Lan, L.; Wen, X.S. Corrosion Rate Simulation and Influence Factors of a Vertical DC Grounding Electrode. IEEE Access 2018, 6, 57230-57238. [CrossRef]

5. Hu, J.; Zeng, R.; He, J.; Sun, W.M.; Yao, J.X. Novel Method of Corrosion Diagnosis for Grounding Grid. In Proceedings of the International Conference on Power System Technology, Perth, WA, Australia, 4-7 December 2000; pp. 1365-1370.

6. Qu, Y.J.; Xu, W.W.; Li, S.L.; Liu, H. Corrosion fault diagnosis for substation's grounding grid based on micro method. In Proceedings of the 2017 China International Electrical and Energy Conference, Beijing, China, 25-27 October 2017; pp. 658-662.

7. Xiao, X.H.; Chen, X.L. Analysis of theory and method about the corrosion as well as the broken point of the grounding grid. J. Chongqing Univ. 2001, 24, 72-75.

8. Zhang, X.L.; Chen, X.L. The technique of the optimization applied in the grounding grid's failure diagnosis of the power plant and substation. High Volt. Eng. 2000, 26, 64-66.

9. Xu, G.; Zhu, Z.H.; Yan, P.B. Optimization Algorithm of Corrosion Diagnosis for Grounding Grid. In Proceedings of the 2nd International Conference on Mechanical and Electronics Engineering (ICMEE 2010), Kyoto, Japan, 1-3 August 2010; pp. 42-46.

10. Liu, J.; Wang, J.X.; Wang, S. An improved fault diagnosis algorithm for grounding grids and evaluation of test schemes. J. Electr. Eng. China 2005, 25, 71-77.

11. Liu, L.Q.; Luo, X.J.; Tao, N.; Wang, K. Grounding Grid Corrosion Diagnosis Based on Large Change Sensitivity. In Proceedings of the Fifth International Conference on Information Assurance and Security, Xi'an, China, 18-20 August 2009; pp. 71-74.

12. Liu, J.; Wang, J.X.; Wang, S. A Corrosion Diagnosis Approach for Grounding Grids Based on TABU Search Algorithm. In Proceedings of the Fifth International Conference on Machine Learning and Cybernetics, Dalian, China, 13-16 August 2006; pp. 1088-1091.

13. Du, J.Y.; Li, N.; Yan, A.J. A Grounding Grid Model of Corrosion Factors Based on Clustering and SVR. J. Inf. Comput. Sci. 2013, 10, 5243-5251. [CrossRef]

14. Huang, Y.H.; Hu, L.H.; Cao, L.N.; Xia, H.F. Research of Grounding Grid Fault diagnosis based on differential evolution algorithm. In Proceedings of the International Conference on Power System Technology (POWERCON 2014), Chengdu, China, 20-22 October 2014; pp. 1497-1502.

15. Zhang, Y.J.; Luo, X.J.; Niu, T.; Liu, L.Q. A new fault diagnosis model for grounding grid based on constrained total least squares algorithm. J. Xi'an Jiaotong Univ. 2010, 44, 110-115.

16. Liu, Y.G.; Shang, L.L.; Leng, D.; Mi, H.W.; Ma, J.P. Corrosion diagnosis of grounding grid based on continuum genetic algorithm. High Volt. Technol. 2016, 42, 1503-1510. 
17. Li, T.Y.; Zheng, Y.H.; Li, L.X.; Yao, G.; Xu, Q.S.; Wang, Y.H. Grounding grids corrosion blocking diagnosis algorithm based on dynamic chaotic PSO. Power Syst. Prot. Control 2011, 39, 78-83.

18. Fan, Y.; Wang, Y.A.; Dong, M.L.; Kou, X.K.; Yao, D.G.; Li, X.; Gao, B.; Irfan, U. A cycle voltage measurement method and application in grounding grids fault location. Energies 2017, 10, 1929.

19. Liu, J.; Ni, Y.F.; Wang, S.Q.; Li, Z.H.; Wang, J.X.; Wang, S. A Novel Approach of Grounding Grid Corrosion Diagnosis. In Proceedings of the IEEE International Conference on Industrial Technology, Chengdu, China, 21-24 April 2008; pp. 1-5.

20. Liu, Y.G.; Xiao, L.S.; Tian, J.H. Optimized Corrosion Diagnosis of Large-Scale Grounding Grid. In Proceedings of the Power \& Energy Society General Meeting, Providence, RI, USA, 25-29 July 2010; pp. 1-6.

21. Zhou, B.; Peng, M.F.; Huang, Q.X.; Jing, J.; Shen, M.E. Grounding grid fault diagnosis based on node tearing and chemical reaction optimization algorithm. Electr. Power Autom. Equip. 2017, 37, 163-168.

22. Wang, S.Q.; Liu, J.; Wang, S.; Li, Z.Z. Grounding Grid Corrosion Diagnosis and Uncertainly Analysis of Branches. J. Comput. 2010, 5, 1289-1295. [CrossRef]

23. Liu, L.Q.; Luo, X.J.; Niu, T. Creditability analysis of grounding grid corrosion diagnosis. Int. J. Appl. Electromagn. Mech. 2010, 33, 1591-1597. [CrossRef]

24. Liu, J.; Ni, Y.F.; Lu, W.; Wang, S.; Li, Z.Z. Influence of Touchable Nodes Deviation on Grounding Grids Corrosion Diagnosis and Its Correction. High Volt. Eng. 2008, 34, 2349-2354.

25. Liu, Y.G.; Leng, D.; Xiao, L.S.; Tian, J.H. Analysis of Influence of Substation Frame on Corrosion Diagnosis of Grounding Grid. J. Chongqing Univ. 2013, 36, 92-109.

26. Liu, Y.G.; Cheng, W.J. Influence of grounding conductor in cable trench of substation on corrosion diagnosis. High Volt. Technol. 2014, 40, 505-512.

27. Gomes, L.V.; de Macedo, E.C.T.; Albuquerque, T.C.; Guedes, E.C.; Junior, G.V.A.; de Castro, M.S.; Freire, R.C.S. Embedded System to Grounding Grid Diagnosis of Energized Substations. In Proceedings of the Instrumentation and Measurement Technology Conference, Graz, Austria, 13-16 May 2012; pp. 796-800.

28. Dawalibi, F. Electromagnetic fields generated by overhead and buried short conductors: Part 2 Ground Networks. IEEE Trans. Power Deliv. 1986, 1, 112-119. [CrossRef]

29. Zhang, B.; Zhao, Z.B.; Cui, X. Diagnosis of breaks in substaiton's grounding grid by using the electromagnetic method. IEEE Trans Magn. 2002, 38, 473-476. [CrossRef]

30. Liu, Y.; Cui, X. Research and application of the system on measuring magnetic field in complicated EMI environment in substation. In Proceedings of the IEEE International Symposium on Electromagnetic Compatibility, Qingdao, China, 23-26 October 2007; pp. 146-149.

31. He, Z.Y.; Hu, H.T.; Huang, W.; Pan, Y.L.; Yang, J.W. A Method of Defect Diagnosis for Integrated Grounding System in High-Speed Railway. IEEE Trans. Ind. Appl. 2015, 51, 5139-5148. [CrossRef]

32. Zhang, P.H.; He, J.J.; Zhang, D.D.; Wu, L.M. A Fault Diagnosis Method for substation grounding grid based on the square-wave frequency domain model. Metrol. Meas. Syst. 2012, 19, 63-72. [CrossRef]

33. Qamar, A.; Umair, M.; Fan, Y.; Uzairm, M.; Kaleem, Z. Derivative method-based orientation detection of substation grounding grid. Energies 2018, 11, 1873. [CrossRef]

34. Qamar, A.; Shah, N.; Kaleem, Z.; Uddin, Z.; Orakzi, F. Breakpoint diagnosis of substation grounding grid using derivative method. Prog. Electromagn. Res. 2017, 57, 73-80. [CrossRef]

35. Liu, K.; Yang, F.; Zhang, S.Y.; Zhu, L.W.; Hu, J.Y.; Wang, X.Y.; Ullah, I. Research on Grounding Grids Imaging Reconstruction Based on Magnetic Detection Electrical Impedance Tomography. IEEE Trans. Magn. 2018, 54, $1-4$.

36. Shao, Y.P.; Mu, M.M.; Zhang, B.; Nie, K.B.; Liao, Q.Q. Corrosion Behavior of Copper-Clad Steel Bars with Unclad Two-End Faces for Grounding Grids in the Red Clay Soil. J. Mater. Eng. Perform. 2017, 26, 1751-1757. [CrossRef]

37. Li, J.; Su, H.; Feng, C.; Xie, D.M.; Li, L.; Li, X.Y.; Meng, H.M. Corrosion behavior of low-carbon Cr micro-alloyed steel for grounding grids in simulated acidic soil. J. Iron Steel Res. Int. 2018, 25, 755-766. [CrossRef]

38. Han, L.; Song, S.Z.; Zhang, X.L.; Li, Y.L.; Qiu, Z.G. Portable grounding grid corrosion electrochemical detection system and its application. Corros. Sci. Prot. Technol. 2009, 21, 337-340.

39. Yang, T.; Peng, M.F.; Hong, H.T. A Practical Method for Detecting the Status of Grounding Grids. In Proceedings of the 2nd International Conference on Industrial Mechatronics and Automation, Wuhan, China, 30-31 May 2010; pp. 270-273. 
40. Law, D.W.; Millard, S.G.; Bungey, J.H. Linear polarization resistance measurements using a potentiostatically controlled guard ring. NDT E Int. 2000, 33, 15-21. [CrossRef]

41. Jiao, J.P.; Zhong, X.; He, C.F.; Wu, B. Experiments on non-destructive testing of grounding grids using SH0. Insight Non-Destr. Test. Cond. Monit. 2012, 54, 375-379.

42. Jiang, Y.M.; Chen, W.G.; Huang, Y.; Chen, H.G. The Application of Ultrasonic Guided Wave in Grounding Grid Corrosion Diagnosis. Int. J. Comput. Electr. Eng. 2013, 5, 313-316. [CrossRef]

43. Rodrigues, N.R.; de Oliveira, R.M.; Carvalho, L.F.; de Almeida, J.F.S. A Method Based on High Frequency Electromagnetic Transients for Fault Location on Grounding Grids. In Proceedings of the International Microwave and Optoelectronics Conference, Rio de Janeiro, Brazil, 4-7 August 2013; pp. 1-5.

44. Yu, C.G.; Fu, Z.H.; Wang, Q.; Tai, H.M.; Qin, S.Q. A Novel Method for Fault Diagnosis of Grounding Grids. IEEE Trans. Ind. Appl. 2015, 51, 5182-5188. [CrossRef]

45. Yu, C.G.; Fu, Z.H.; Hou, X.Z.; Heng, M.T.; Su, X.F. Break Point Diagnosis of Grounding Grids Using Transient Electromagnetic Apparent Resistivity Imaging. IEEE Trans. Power Deliv. 2015, 30, 2485-2491. [CrossRef]

46. Yu, C.G.; Fu, Z.H.; Wu, G.L.; Zhou, L.Y.; Zhu, X.G.; Bao, M.H. Configuration detection of substation grounding grid using transient electromagnetic method. IEEE Trans. Ind. Electron. 2017, 64, 6475-6483. [CrossRef]

47. Li, X.; Yang, F.; Ming, J.; Jadoon, A.; Han, S. Imaging the corrosion in grounding grid branch with inner-source electrical impedance tomography. Energies 2018, 11, 1739. [CrossRef]

48. Wu, X.; Han, X. A RADAR detection system with random noise transmitting wave for power grounding grid. Front. Artif. Intell. Appl. 2017, 299, 410-415.

49. Wang, Z.M.; Guo, Y.L.; Fu, Q.; Pan, L. A Detection Radar for Grounding Grid. In Proceedings of the 2nd IEEE Advanced Information Management, Communicates, Electronic and Automation Control Conference, Xi'an, China, 25-27 May 2018; pp. 932-935.

50. Charalambous, C.A.; Demetriou, A.; Kokkinos, N.D. Impact of Photovoltaic-Oriented DC Stray Current Corrosion on Large-Scale Solar Farms' Grounding and Third-Party Infrastructure: Modeling and Assessment. IEEE Trans. Ind. Appl. 2015, 51,5421-5430. [CrossRef]

51. Fu, Z.H.; Song, S.Y.; Wang, X.J.; Li, J.Q.; Tai, H.M. Imaging the Topology of Grounding Grids Based on Wavelet Edge Detection. IEEE Trans. Magn. 2018, 54, 1-8. [CrossRef]

52. Chen, L.; Peng, M.F.; Miao, W.H. Application of Information Fusion in Grounding Network Breakpoint Diagnosis. J. Power Syst. Autom. 2010, 22, 25-30.

53. Du, J.Y.; Wang, L.C. Research on corrosion monitoring system of grounding grid based on cloud platform. In Proceedings of the 32nd Youth Academic Annual Conference of Chinese Association of Automation, Hefei, China, 19-21 May 2017; pp. 1255-1259. 